\title{
Semiotik
}

Band $37 \cdot$ Heft 1-2 (2015)

Seite 55-79

Stauffenburg Verlag Tübingen

\section{Gibt es ein Lachen der Schadenfreude?*}

\author{
Jennifer Hofmann und Willibald Ruch, Universität Zürich
}

\begin{abstract}
Summary. At the beginning of the 20th century, authors concerned with Ausdruckspsychologie ("expression psychology") described the existence of a specific visual and auditory morphological pattern of schadenfreude laughter. They were putting enormous efforts in visual and verbal illustrations of several laughter types, but those ideas have been widely neglected in modern research. Preceding studies on the frequency and intensity of smiling and laughter in 16 positive emotions showed that not only amusement, but also schadenfreude elicited laughter and more intense facial displays of smiling and laughter. Thus, the current study investigated three hypotheses on the facial expression of schadenfreude in 17 recalled schadenfreude events. The results showed that schadenfreude went along with laughter very often (laughter occurred in over $70 \%$ of the recalled schadenfreude memories). The facial expressions of smiling and laughter in schadenfreude were frequently accompanied by markers of display regulation and dampening. Therefore, the joyful expression of schadenfreude in the face (Duchenne display) is often distorted by regulation or dampening actions. This is probably due to the social desirability of not laughing at others' misfortunes.
\end{abstract}

Zusammenfassung. Bereits zu Beginn des 20. Jahrhunderts beschrieben die Ausdruckspsychologen sowohl einen spezifischen Gesichtsausdruck als auch eine besondere Lautbildung, die nur dem Lachen der Schadenfreude zu eigen sein sollte. Leider wurden diese Ideen bislang nur unzureichend durch gezielte Untersuchungen weiterverfolgt. Vorarbeiten zur vorliegenden Studie, in denen die Häufigkeit und Intensität von Lächeln und Lachen in 16 positiven Emotionen untersucht wurden, zeigten, dass nicht nur Erheiterung systematisch mit Lachen und intensiveren mimischen Reaktionen einhergeht, sondern auch Schadenfreude. Aufbauend auf diesen Ergebnissen testete die vorliegende Studie drei Hypothesen zum mimischen Ausdruck von Schadenfreude in 17 erinnerten Schadenfreude-Erlebnissen. Die Resultate zeigen, dass Schadenfreude sehr oft mit Lachen einherging (in $70 \%$ der Schadenfreude-Erlebnisse) und darüber hinaus die mimischen Ausdrücke oft durch Marker der Regulierung/Dämpfung des Ausdruckes gekennzeichnet waren. Während Schadenfreude also durch den für Freude typischen Gesichtsausdruck gezeigt wird (Duchenne-Display), findet im sozialen Kontext oft eine sichtbare Abschwächung (Regulation) oder ein Verbergen des Ausdrucks statt. Dies ist womöglich darauf zurückzuführen, dass es sozial nicht akzeptabel ist, über den Schaden anderer öffentlich zu lachen. 


\section{Einleitung}

\subsection{Die Morphologie "des Lachens“}

Angesichts des Umstands, dass Lachen als eines der hervorstechendsten nonverbalen Kommunikationsmittel gilt, ist es erstaunlich, wie wenig über dieses Ausdrucksverhalten bekannt ist (siehe Hofmann 2014; Ruch und Ekman 2001). Die Frage, wie viele Arten des Lachens es gibt, die auch morphologisch unterschieden werden können, ist noch unbeantwortet (siehe Ruch, Hofmann und Platt 2013). Auch gibt es keine Einigung darüber, wie die verschiedenen Formen des Lachens klassifiziert werden können. Während lexikalische Untersuchungen der deutschen Sprache ergeben, dass Lachen mit über 1000 beschreibenden oder qualifizierenden Wörtern einhergeht (siehe Ruch und Wagner in diesem Heft), ist unklar, wie viele dieser Lachtypen sich auch eines distinkten Ausdrucksmusters bedienen, das sich mit hinreichender Genauigkeit anhand der Stimme, des Gesichts oder der Körperbewegungen identifizieren lässt.

Der Großteil des Forschungskanons zum Lachen konzentrierte sich auf die Erforschung des stimmlichen Ausdrucksmusters, sowohl auf der Seite der Lachenden (d.h. in der Enkodierung) als auch auf der Seite der Empfänger (d.h. in der Dekodierung). ${ }^{1}$ Dabei zeigte sich, dass die Phonation beim Lachen inter- und intra-individuell variiert, aber nur vokalisiertes Lachen (also „ha-ha-ha“ im Vergleich zu „h-h-h“) unabhängig vom Auslöser als positiver und freundlicher wahrgenommen wird als nicht-vokalisiertes Lachen. Unterdrücktes Lachen, bei dem die lachende Person zum Beispiel die Lippen zusammenpresst, führt beispielsweise dazu, dass das Lachen nur durch eine forcierte Ausatmung hörbar wird. An diesem Beispiel wird deutlich, wie sich die Mimik und der stimmliche Ausdruck gegenseitig beeinflussen. Je nach Öffnung des Mundes klingt das Lachen anders. Daher ist es also wichtig, das Lachen nicht nur als ein akustisches Phänomen zu studieren, sondern auch dessen mimischen Ausdruck zu beachten.

Zum jetzigen Zeitpunkt ist allerdings vergleichsweise wenig über den mimischen Ausdruck des Lachens bekannt (z.B. Ruch, Hofmann und Platt 2013). In den meisten vorliegenden Studien wurde das sogenannte „Duchenne-Lachen" untersucht (siehe Ruch und Ekman 2001), das sich durch ein spezifisches Zusammenspiel von mimischen Markern zusammen mit der lachtypischen Lautbildung auszeichnet.

Wie die Forschung zum Lächeln zeigte, geht das Empfinden von Freude in der Regel mit dem Ausdruck eines bestimmten Lächelns einher, dem Duchenne-Lächeln (siehe Ekman 1989; Ekman, Davidson und Friesen 1990; Frank und Ekman 1993). Diese mimische Konfiguration besteht sowohl aus der Anspannung des großen Jochbeinmuskels (m. zygomaticus major), der die Mundwinkel nach oben und hinten zieht, als auch der zeitgleichen und symmetrischen Anspannung des Augenringmuskels (m. orbicularis oculi pars orbitalis). Dieses Duchenne-Lächeln lässt sich von 14 bis 18 weiteren Formen von Lächeln (siehe Bänninger-Huber 1996; Ekman 1985) 
qualitativ abgrenzen. So unterscheidet sich das Duchenne-Lächeln von anderen (z.B. gestellten Formen) des Lächelns im Timing der mimischen Veränderungen, in der Symmetrie (siehe Ekman u.a. 1981; Indersmitten und Gur 2003), im dynamischem Verlauf (Ambadar u.a. 2009; Hess und Kleck 1990; Krumhuber und Kappas 2005; Krumhuber u.a. 2007; Schmidt u.a. 2006, 2009), im Auftreten des Lächelns während des Redens, im zeitgleichen Stattfinden weiterer motorischer Verhaltensmuster (Ekman und Friesen 1982) und in der Intensität der mimischen Marker (siehe Krumhuber und Manstead 2009). Das Duchenne-Lächeln als wiederkehrende und aussagekräftige Form des mimischen Ausdrucks der Freude wurde öfter in Frage gestellt. ${ }^{2}$ Jedoch konnte eine Vielzahl von Studien zeigen, dass Duchenne-Lächeln dann häufiger vorkommt, wenn Personen Freude empfinden (gemessen an Selbstberichten), humorvolle Cartoons ansehen und diese auch als witzig einschätzen sowie wenn sie positive Emotionen erleben (vgl. Ekman, Friesen und Ancoli 1980; Ekman, Davidson und Friesen 1990; Harris und Alvarado 2005; Keltner und Bonanno 1997; Keltner u.a. 2003; Matsumoto u.a. 2008; Ruch 1993, 1995 und 1997). Der Begriff „positive Emotion“ wird im Folgenden so verstanden, dass diese Emotion mit einem angenehmen Gefühl, einer positiven Valenz einhergeht - es geht dabei nicht um eine Wertung der Emotion.

Aufbauend auf der Forschung zum Duchenne-Lächeln als mimischen Marker von Freude konnten Studien aus dem Bereich der Humorforschung unter anderem auch zeigen, dass Erheiterung mit einem Lachen einherging, das mimisch dieselben Marker enthielt wie das Duchenne-Lächeln. In der Folge wurde oftmals das Duchenne-Lachen untersucht, bestehend aus derselben mimischen Konfiguration (aber mit zusätzlich offenem Mund und gesenktem Kiefer) und der für Lachen typischen Lautbildung (siehe Ruch und Ekman 2001). Dessen ungeachtet gibt es Hypothesen, dass beim Lachen auch andere mimische Marker auftreten (siehe Hofmann 2014). Sumitsuji (1967) leitete mit Nadelelektroden das elektrische Potential direkt aus den Gesichtsmuskeln lachender Personen ab und fand, dass auch weitere Muskeln (die Teil des Duchenne-Displays sind) Aktivierungen zeigen. Es ist also denkbar, dass Lachen auch noch andere mimische Aktionen beinhaltet. Ungeklärt bleibt dabei auch, ob sich die Charakteristika im Gesichtsausdruck je nach Auslöser, d.h. zwischen qualitativ unterschiedlichen Arten des Lachens unterscheiden. Dies würde zu zusätzlichen Unterschieden in der Mimik führen, abhängig vom Stimulus, der das Lachen auslöst.

„Das“ Lachen wurde bislang hauptsächlich im Kontext von Erheiterung als Auslöser untersucht, obwohl es vielerlei Hinweise darauf gibt, dass mehrere andere Emotionen typischerweise Lachen auslösen. Eine grundlegende Annahme ist hierbei, dass verschiedene emotionale Auslöser zu unterschiedlichen Arten des Lachens führen, die sich auch anhand einer eigenen Morphologie auseinanderhalten lassen sollten. Welche emotionalen Auslöser bieten sich also an, wenn untersucht werden soll, ob es ein Lachen jenseits des durch Erheiterung ausgelösten Duchenne-Lachens gibt? Eine (positive) Emotion, die bereits mit einem spezifischen Lachen in Verbin- 
dung gebracht wurde, ist Schadenfreude. Schadenfreude hat eine positive Valenz (zumindest zu einem Teil) und geht daher auch mit einer angenehmen Erlebensqualität einher. Dieser Fährte folgend soll in diesem Artikel daher der Frage nachgegangen werden, ob es ein Lachen der Schadenfreude gibt, welches mit einem spezifischen mimisch-morphologischen Ausdruckmuster einhergeht.

\subsection{Die Schadenfreude}

Im Rahmen der vorliegenden Studie wird Schadenfreude als Emotion verstanden. Sie bezeichnet die Freude, die jemand empfindet, wenn einer beneideten (Smith u.a. 1996; Van Dijk u.a. 2005), einer ungeliebten (Hareli und Weiner 2002) oder einer Ärger auslösenden (Feather und Sherman 2002) Person oder Gruppe ein Missgeschick widerfährt oder sie eine peinliche Situation erlebt. Die Stärke der empfundenen Schadenfreude hängt davon ab, wie sehr die schadenfrohe Person empfindet, dass die geschädigte Person das Missgeschick verdient hat, wie selbstbewusst erstere Person ist, wie sehr sie sich bedroht fühlt und wie neidisch sie ist (Brigham u.a. 1997; Feather 2008; Feather und Sherman 2002; van Dijk u.a. 2005; van Dijk u.a. 2011). Eine Vielzahl von Arbeiten wurde bereits den auslösenden und moderierenden Faktoren der Schadenfreude gewidmet. Dabei wurde die Stärke der empfundenen Emotion zumeist anhand von Selbstberichten erfasst.

Ekman (2003) führte Schadenfreude als eine der 16 Facetten positiver Emotionen auf. Er ging davon aus, dass alle 16 positiven Emotionen mimisch durch das Duchenne-Lächeln ausgedrückt werden und sich etwaige Unterschiede im akustischen Ausdruck finden lassen könnten. Hofmann, Ruch und Platt (2012) stellten fest, dass bis dahin vor allem Forschung zum akustischen Ausdruck von Schadenfreude (siehe Szameitat u.a. 2009, 2010; Wildgruber u.a. 2013) berichtet und der durch Schadenfreude ausgelöste Gesichtsausdruck dagegen wenig untersucht wurde. Drei Studien erfassten neben der subjektiv eingeschätzten Stärke der empfundenen Schadenfreude auch die mimischen Reaktionen (Boecker u.a. 2015; Cikara und Fiske 2013; Porter u.a. 2014). In einer vierten Studie wurden die mimischen Reaktionen als Antwort auf Szenarien gemessen, in denen Schadenfreude beschrieben wurde (Platt u.a. 2013). Die Ergebnisse der vier Studien sollen zunächst genauer beschrieben werden.

Porter und Kollegen (2014) lösten bei ihren Versuchspersonen Schadenfreude aus und erfassten die mimischen Reaktionen der Probanden, während diese vier Bilder sahen, auf denen einer Person ein Missgeschick widerfuhr. Sie fanden dabei, dass Versuchspersonen sowohl Lächeln als auch Lachen zeigten, beides gemessen an der Aktivierung des großen Jochbeinmuskels, der auch für den Duchenne-Ausdruck charakteristisch ist (zuzüglich einer hörbaren Vokalisation im Falle eines Lachens), zogen aber keine weiteren mimischen Indikatoren zur Messung hinzu (wie zum 
Beispiel den Augenringmuskel). So zeigte diese Studie zwar, dass Schadenfreude Lachen und Lächeln als nonverbale Zeichen auslöste, gab aber keinen Aufschluss darüber, welche weiteren mimischen Marker neben der Aktivierung des Jochbeinmuskels auftraten; d.h., ob es sich um ein „echtes" Lächeln der Freude handelt.

Cikara und Fiske (2013) setzten Elektromyographie (EMG) zur Messung der Aktivierung des großen Jochbeinmuskels in Reaktion auf Szenarien ein, bei denen auch Selbstberichte von Schadenfreude vorlagen. Sie konnten dabei demonstrieren, dass die Anspannung des Jochbeinmuskels mit der Stärke der empfundenen Schadenfreude anstieg.

Boecker und Kollegen (2015) zeigten, dass sich die mimischen Ausdrücke der Freude und Schadenfreude nicht in der Aktivierung von vier mit EMG gemessenen Muskeln unterscheiden. Sie bedienten sich einer Messung der Aktivierung der mm. zygomaticus major (großer Jochbeinmuskel), orbicularis oculi (Augenringmuskel), corrugator supercilii (senkt die Augenbrauen oblique zur Nase hin) und frontalis supercilii (Stirnmuskel) bei 32 fußballinteressierten Männern. Es zeigte sich, dass in Spielsituationen, die Schadenfreude auslösen sollten, gleichermaßen wie in solchen, die dafür gedacht waren, Freude hervorzurufen, sowohl eine Zunahme in der Aktivierung des $\mathrm{m}$. zygomaticus major und $\mathrm{m}$. orbicularis oculi als auch eine Senkung des Muskeltonus im $\mathrm{m}$. corrugator supercilii zu verzeichnen war (in Schadenfreude-Situationen nahm der Muskeltonus des letzteren stärker ab als in Freude-Situationen). Duchenne-Lächeln bei Entspannung des „Stirnrunzlers“ wurde bei vielen Studien zur Freude gefunden. Die Personen wurden alleine getestet, es gab also keine Verzerrung durch soziale Erwünschtheit (d.h. der Tendenz, sich so zu verhalten, wie es den sozialen Regeln und Normen einer Kultur oder Gruppe entspricht). Diese Resultate sprechen für Ekmans Hypothese, dass alle Facetten von Freude durch Lächeln ausgedrückt werden, es war aber keine morphologische Trennung der Ausdrücke anhand der gemessenen vier Muskeln möglich.

In einer Studie zu 16 positiven Emotionen konnten Platt und Kollegen (2013) zeigen, dass mindestens fünf positive Emotionen auch mit Lachen einhergingen: Erheiterung, Schadenfreude, Erleichterung, taktile Freude und freudige Verwunderung. All diese Emotionen gingen mit DuchenneLachen einher, wenn Versuchspersonen davon berichteten, was sie bei verschiedenen Szenarien empfinden würden, die diese Emotionen auslösen sollten. Interessanterweise zeigten die Ergebnisse, dass Schadenfreude sehr oft mit Lachen einherging.

Allen Studien ist gemeinsam, dass Schadenfreude Lächeln und Lachen auslöste, welche die Aktivierung des Jochbeinmuskels beinhaltet. Zwei der Studien konnten zeigen, dass Schadenfreude mit dem DuchenneLächeln und Lachen einhergeht. Leider hat keine der Studien eine größere Anzahl mimischer Marker in die Ergebnisse miteinbezogen: Mittels Gesichts-EMG maßen Boecker und Kollegen (2015) die Aktivierung von vier Innervationsregionen, Cikara und Fiske (2013) die von zwei. Porter und Kollegen (2014) kodierten lediglich die Aktivierung des Jochbeinmus- 
kels, und Platt und Kollegen (2013) beschränkten sich auf die Kodierung von Duchenne-Lächeln und Lachen. Während also außer Frage steht, dass durch Schadenfreude Lächeln und Lachen ausgelöst werden, bleibt offen, ob dieses Lächeln und Lachen sich morphologisch von dem von anderen Arten von Freude ausgelösten unterscheiden lässt, d.h., ob das "Lachen der Schadenfreude" eine spezifische mimische Qualität hat.

\subsection{Fast vergessenes Wissen aus der Ausdruckspsychologie}

Während nur wenige Forscher aus der jüngeren Vergangenheit sich dem morphologischen Ausdruck des Schadenfreude-Lachens angenommen haben, waren Vertreter der Ausdruckspsychologie ${ }^{3}$ auf diesem Gebiet äußerst aktiv und haben dem Lachen der Schadenfreude eine bestimmte Qualität zugewiesen, welche im mimischen und stimmlichen Ausdruck identifizierbar ist. In einer Aufarbeitung der Schriften historischer Autoren haben wir (Ruch, Hofmann und Platt 2013) die damaligen Annahmen zum Ausdruck des Lachens der Schadenfreude systematisiert und getestet. Drei Autoren nominierten ein Lachen der Schadenfreude und lieferten dazu jeweils eine verbale Beschreibung sowie eine Zeichnung bzw. Photographie (siehe Abb. 1).

Schadenfreude-Lachen (Nomination durch Autoren)

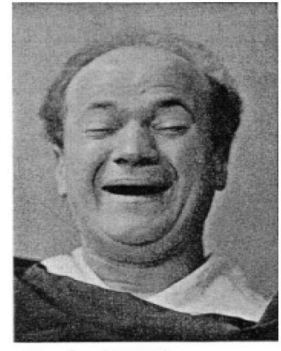

Borée (1899)

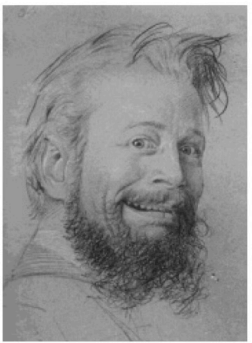

Rudolph (1903)

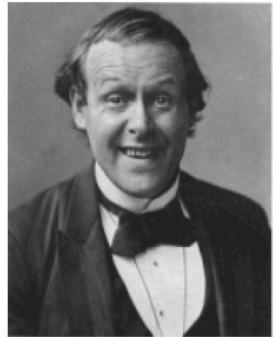

Huter (1925)

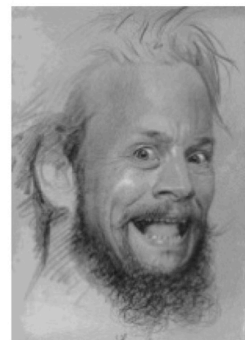

Rudolph (1903)

Schadenfreude-Lachen (Nomination durch Dekodierung)

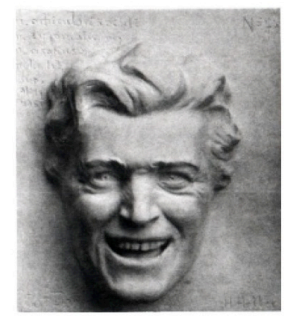

Heller (1902)

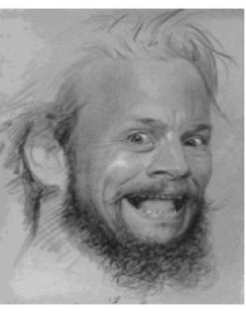

Rudolph (1903)

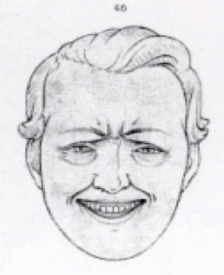

Piderit (1867)

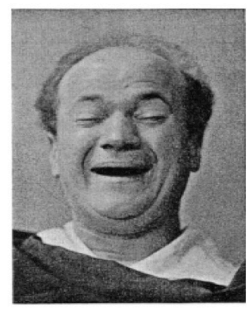

Borée (1899)

Abb. 1: Visuelle Repräsentationen von Schadenfreude-Lachen, gruppiert nach Art der Nomination (oben Klassifikation durch Originalautor, unten Zuordnung aufgrund von Dekodierung). 
In Abb. 1 sind in der oberen Reihe die Portraits des Schadenfreude-Lachens zu sehen, welche durch Huter (1925), Rudolph (1913) und Borée (1899) beschrieben wurden.

Die Kodierung der oberen Reihe von Abb. 1 durch Ruch und Mitarbeiter (2013) zeigte, dass in allen Darstellungen von Lachen eine Aktivierung des Jochbeinmuskels ersichtlich und der Mund geöffnet, der Kiefer oft auch gesenkt ist. Zudem zeigen sich auf allen Illustrationen Falten auf der Stirn. Abgesehen von diesen Gemeinsamkeiten lässt sich in den visuellen Darstellungen kein einheitliches Muster von mimischen Markern ableiten: Huters (1925) Illustration zeigt eine Aktivierung des Jochbeinmuskels mit geöffnetem Mund und gesenktem Kiefer. In der oberen Gesichtshälfte sind Falten auf der Stirn erkennbar (m. frontalis), und die Augenlider sind angespannt. Rudolph (1913) lieferte zwei Illustrationen. In beiden kann wiederum eine Aktivierung des Jochbeinmuskels mit geöffnetem Mund und gesenktem Kiefer gesehen werden. Der Augenringmuskel ist angespannt und die Augenlider sind geweitet. Die beiden Illustrationen unterscheiden sich darin, dass in der einen zusätzlich die Unterlippe nach oben geschoben und das Kinn angehoben wird, als auch die Lippen horizontal angespannt sind (m. risorius). In der Illustration des intensiven Schadenfreude-Lachens sind im oberen Gesichtsteil zusätzlich sowohl eine Anspannung der Augenlider als auch ein Zusammenziehen der Augenbrauen sichtbar. Borées (1899) Illustration unterscheidet sich am meisten von den anderen, nicht nur in der Qualität der mimischen Marker, sondern auch in der Intensität dieser Marker und der Symmetrie/Lateralität. Die Mundwinkel scheinen auf den beiden Gesichtshälften simultan von gegenläufigen Muskelaktivierungen betroffen zu sein: Auf der rechten Seite sind die Mundwinkel vertikal nach unten gezogen, was der Aktivierung des Jochbeinmuskels entgegenwirkt. Zudem ist die rechte äußere Augenbraue angehoben. Auffällig ist zudem, dass die Stirn tiefe Falten aufweist und die Augenlider stark angespannt sind, im Vergleich zum lediglich mittelmäßig intensiven Lächeln (siehe Ruch u.a. 2013).

Es ist auffällig, dass die Öffnung des Mundes in keinem der Fälle sehr groß dargestellt wurde, was darauf hindeutet, dass keines der Schadenfreude-Lachen als unreguliertes, uninhibiertes Lachen verstanden wurde. Zudem war ersichtlich, dass mimische Marker dargestellt wurden, welche auf eine Regulierung/Abschwächung ${ }^{4}$ des Lachens hindeuten. So zum Beispiel das asymmetrische Herunterziehen der Mundwinkel bei Borée, das Hochheben des Kinns und Anspannen der Lippen bei Rudolph. Diese Darstellungen implizieren also, so wurde geschlussfolgert, dass Schadenfreude nicht frei gezeigt werden kann (Ruch, Hofmann und Platt 2013).

In der unteren Reihe der Abb. 1 sind die Ausdrücke des Lachens dargestellt, welche unabhängig von ihrer Originalklassifikation durch die historischen Autoren als Schadenfreude-Lachen von naiven Versuchsteilnehmenden wahrgenommen wurden. Erstaunlicherweise wurde keine der intendierten Manifestationen des Schadenfreude-Lachens als solche dekodiert. In den Nominationen durch die naiven Versuchspersonen ist ersichtlich, dass alle Lachen ein Zusammenziehen der Augenbrauen beinhalte- 
ten. Dieser Marker wurde zwar von Darwin (1872) als Marker der Intensität eines Lachens postuliert, es gibt aber Hinweise, dass der m. corrugator mit der Wahrnehmung von Boshaftigkeit und Schadenfreude in Verbindung gebracht wird, wenn Lachen durch statische Stimuli (d.h. Bilder) dargestellt wird, im Vergleich zu bewegten Bilder in Form von Videoclips (siehe Hofmann 2014). Die Nominationen der Versuchspersonen beinhalten sowohl Elemente der Freude (Jochbeinmuskelaktivierung) als auch mimische Aspekte, die mit negativen Emotionen assoziiert sind (Nasen rümpfen und Stirn runzeln/Augenbrauen zusammenziehen).

\subsection{Hypothesen zum Ausdruck der Schadenfreude}

Aus den Analysen der historischen Illustrationen und deren Bewertung durch naive Versuchspersonen sowie aus dem Studium der neueren Forschung haben wir drei Hypothesen zum Ausdruck der Schadenfreude abgeleitet. Diese drei Hypothesen ergeben sich aus unterschiedlichen theoretischen Annahmen zur Struktur der Emotion Schadenfreude und deren Ausdruck in sozialen und nicht sozialen Bedingungen. Im Folgenden werden diese drei Hypothesen skizziert.

\subsubsection{Hypothese 1}

Schadenfreude wird als eine Mischung zweier Emotionen, bestehend aus Freude und einer negativen Emotion wie z.B. Missgunst, Neid oder Ärger verstanden (Smith u.a. 1996; Hareli und Weiner 2002; Feather und Sherman 2002; Szameitat u.a. 2009). Folglich sollte sich die Schadenfreude im Gesicht auch aus einer Mischung von Markern der Freude (Lächeln und Lachen) und Markern der negativen Emotion (z.B. Zusammenziehen der Augenbrauen) zeigen. Wenn man die Dekodierungen der historischen Illustrationen betrachtet, zeigt sich, dass einige der als Schadenfreude dekodierten Bilder tatsächlich Marker von negativen und positiven Emotionen aufweisen. Da es sich bei den historischen Illustrationen jedoch um gestellte Ausdrücke handelt, kann nicht mit Sicherheit geschlossen werden, dass die Ausdrücke auch bei natürlicher Auslösung ähnlich sein würden.

\subsubsection{Hypothese 2}

Schadenfreude wird als eine Facette von Freude (siehe Ekman 2003) verstanden und wird daher auch durch entsprechende Freude-Marker, d.h. durch Duchenne-Lächeln und Lachen, ausgedrückt. Ekman (2003) beschreibt, dass die Schadenfreude wahrscheinlich in sozialen Situationen unterdrückt oder maskiert wird, da es sozial nicht akzeptabel ist, über das Unglück anderer zu lachen. Dieser Annahme folgend würde die Schadenfreude mimisch also durch Freude ausgedrückt (Lächeln, Lachen), welche aber im sozialen Kontext entweder gedämpft, maskiert oder reguliert wird. Es 
ist daher anzunehmen, dass Lächeln und Lachen durch sogenannte „smile controls“ (Keltner 1995) beeinflusst werden. Unter „smile controls“ wird das Auftreten von Regungen verstanden, welche das Lächeln und Lachen beeinflussen und verändern (Ekman 1990). Dabei können Bewegungen auftreten, welche der Aufwärtsbewegung des Jochbeinmuskels (m. zygomaticus major) entgegenwirken, die das Lächeln verundeutlichen, oder eine Kombination von beidem (Keltner 1995). Am häufigsten treten Aktivitäten der $\mathrm{mm}$. caninus, buccinator, triangularis und mentalis auf (Keltner 1995). Dynamische Unterschiede bei reguliertem Lächeln und Lachen (im Vergleich zu unreguliertem) zeigen sich in der zeitlichen Entfaltung des Ausdrucks, der Dauer und der Geschwindigkeit der Mundwinkelbewegungen, den Asymmetrien in den Mundwinkelbewegungen und in der Kopfhaltung (Ambadar u.a. 2009). Da dynamische Aspekte jedoch schwieriger manuell (z.B. Video) zu erfassen sind als statische, werden sie in Studien häufig nicht berücksichtigt (Ambadar u.a. 2009). Abb. 2 zeigt einige Beispiele, wie das Schadenfreude-Lachen aussehen könnte, indem die Person zugleich versucht, das Lachen zu regulieren oder abzuschwächen.

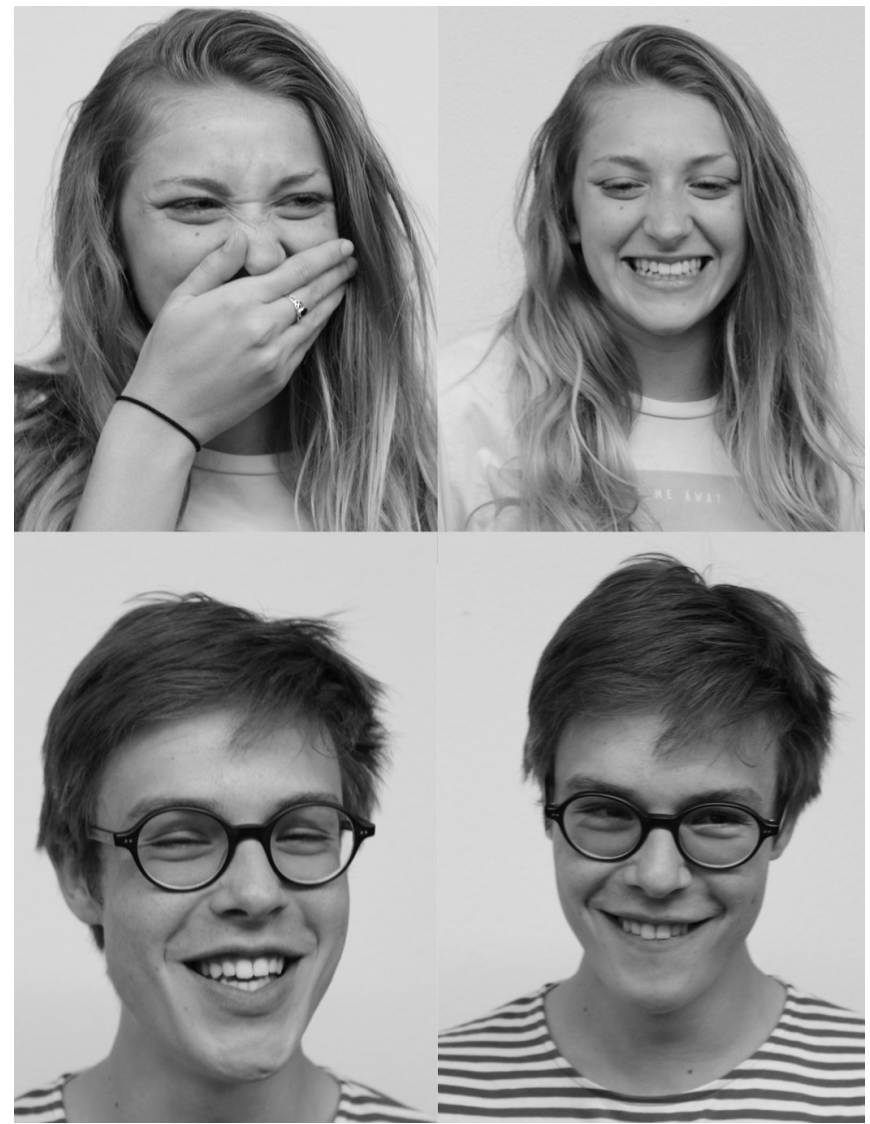

Abb. 2: Beispiele von reguliertem Lachen der Schadenfreude. 
Auf Abb. 2 sieht man oben links, wie die Person während des Lachens mit der Hand den Mund bedeckt. Durch das Verstecken des Mundes ist der Hauptmarker der Freude - das Lächeln/Lachen - nicht mehr sichtbar. So wird die Freude über den Schaden einer anderen Person oder Gruppe „versteckt". Auf dem Bild oben rechts sieht man, wie die Person den Blick nach unten abwendet. Auch die Unterlippen sind in diesem Beispiel horizontal zur Seite gezogen, welches die Aktion des Jochbeinmuskels beeinflusst. Diese beiden Marker deuten darauf hin, dass die Person das Lachen zu dämpfen versucht. Auf dem Bild unten links hat die Person während des Lachens die Augen geschlossen, unten rechts beißt sie sich auf die Lippen - beide Aktionen beeinflussen das ungehemmte Ausdrücken des Lachens. Obwohl alle Beispiele unterschiedliche mimische Marker enthalten, deuten sie auf eine Regulation des Lachens hin.

In der nächsten Bilderserie in Abb. 3 sind neben einem neutralen Gesicht (ganz oben links) und einem frei ausgedrückten Lachen (ganz oben rechts) verschiedene Varianten des Lachens zu sehen, welche abgeschwächt wurden.

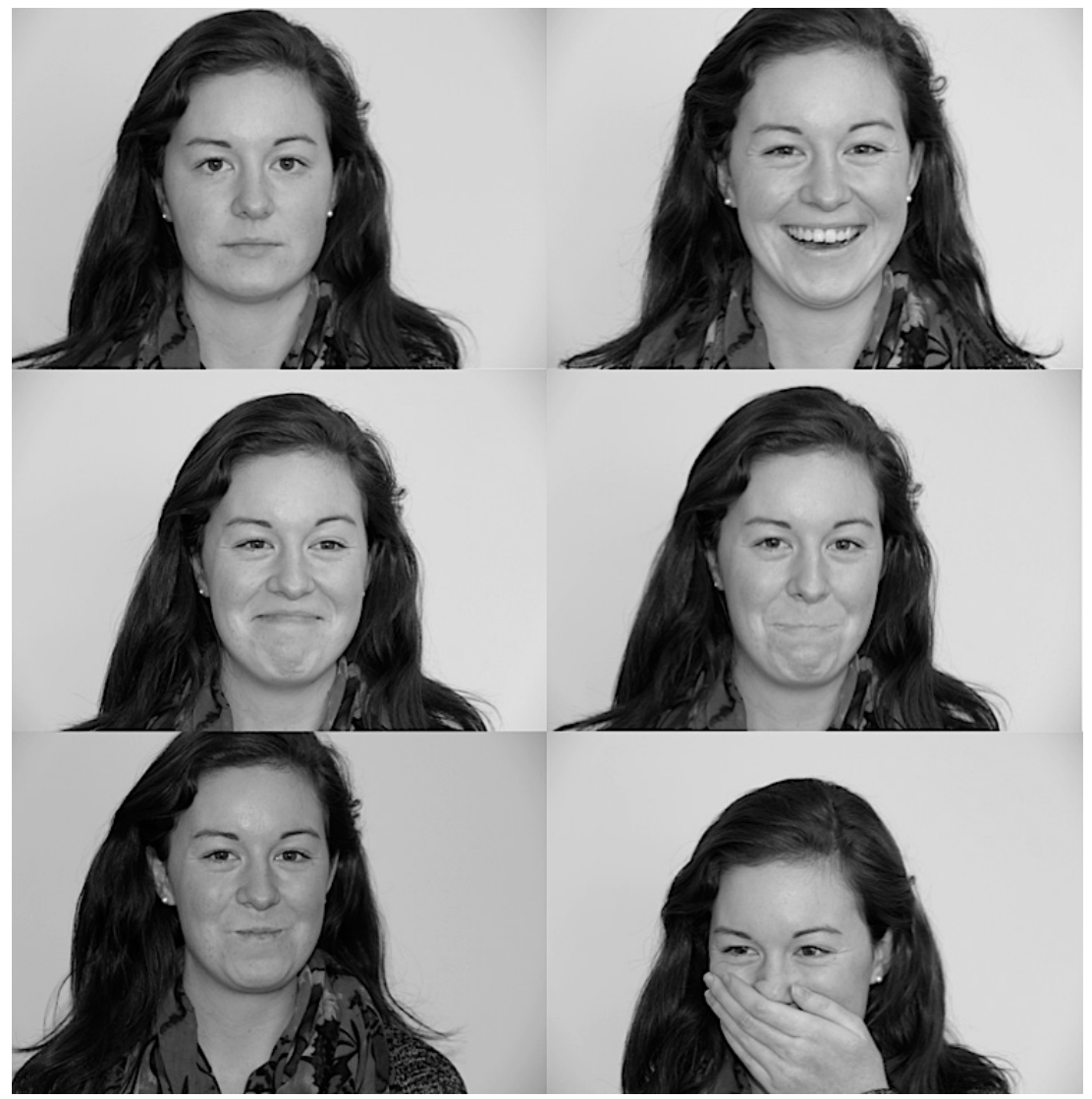

Abb. 3: Weitere Beispiele von reguliertem Lachen der Schadenfreude. 
Abb. 3 zeigt, wie verschiedene mimische Marker die Wahrnehmung der Anspannung des Jochbeinmuskels verzerren. Diese mimischen Marker umfassen die Aktivierung des m. risorius, m. levator anguli oris (auch bekannt als Caninus Muskel) und den Buccinatormuskel. Gemäß der Hypothese 2 sollte das Lachen der Schadenfreude also sowohl die Aktivierung des Jochbeinmuskels enthalten als auch mimische Marker, die das Lachen dämpfen, wobei verschiedene Kombinationen möglich sind. Dabei ist wichtig, dass der spontane Ausdruck des Lachens durch die lachende Person selbst gehemmt wird, um die Freude am „Schaden anderer“ zu vertuschen. Hypothese 2 ist daher insbesondere relevant in Kontexten, in denen die lachende Person nicht alleine ist. Es ist fraglich, ob die Person das Lachen auch regulieren würde, wenn sie alleine ist (denkbar ist dies durchaus, z.B. aufgrund der Annahme eines impliziten Publikums; siehe Fridlund 1991).

\subsubsection{Hypothese 3}

Schadenfreude wird als „soziale Emotion“ verstanden, und es wird angenommen, dass soziale Emotionen nicht mit spezifischen Ausdrucksmustern einhergehen (siehe Bauminger 2004; Dvash und Shamay-Tsoory 2011). Dies impliziert, dass Schadenfreude-Lächeln und -Lachen sich nicht von anderen Arten des Lächelns und Lachens unterscheiden lassen und der Kontext für die korrekte Dekodierung des Ausdruckes notwendig ist. Dieser Tradition (dass nonverbale Ausdrücke soziale Signale sind, die mit gleichem Muster je nach Kontext ähnlich aussehen) folgend, könnte dasselbe Lächeln oder Lachen Empathie, Freude, oder Schadenfreude ausdrücken - je nach Kontext. Folgerichtig sollte sich das Lächeln und Lachen der Schadenfreude nicht von den Ausdrücken anderer positiver Emotionen unterscheiden. Paulus und Wentura (2014) zeigten, dass dasselbe Signal (ein auf einer Fotografie dargestelltes Lächeln) einen anziehenden - oder abweisenden Charakter haben konnte, je nachdem in welchem Kontext es präsentiert wurde. Folglich sollte das Lächeln und Lachen der Schadenfreude morphologisch nicht vom Lächeln und Lachen der Freude unterscheidbar sein. Daher könnte das Lachen der Schadenfreude aus dem DuchenneLachen ohne zusätzliche mimische Marker bestehen.

\subsection{Die vorliegende Untersuchung}

In der vorliegenden Untersuchung bauen wir auf Daten aus einer naturalistischen Gruppenkonversationsstudie auf (Hofmann u.a. 2014). Diese Studie untersuchte das Auftreten von Lächeln und Lachen in 16 positiven Emotionen (basierend auf den Annahmen von Paul Ekman). Die Personen wurden dabei in Gruppen von drei bis vier Personen gebeten, Erinnerungen an 16 verschiedene positive Emotionen wiederzugeben. Dabei wurden die Personen gefilmt und ihre mimischen Reaktionen beim Wiedererleben der 
Gefühle kodiert. Zur Kodierung der mimischen Marker wurde das Facial Action Coding System (FACS; Ekman, Friesen und Hager 2002) verwendet. Zusätzlich wurden Lachvokalisationen kodiert: Deren Vokalisation (oder Phonation: ja vs. nein) und die Länge der Segmente (ein Segment vs. mehrere Segmente; „ha“ vs. "ha-ha-ha“). In einem ersten Schritt wurden alle Ausdrücke des Lächelns und Lachens kodiert, die in Reaktion auf eine Erinnerung auftraten, die klar an eine der positiven Emotionen gekoppelt war. Ziel war es, herauszufinden, ob alle 16 positiven Emotionen mit Lächeln und Lachen einhergehen.

Unsere ersten Resultate zeigten, dass Erheiterung die intensivsten mimischen Ausdrücke von Lächeln und Lachen evozierte und in über $70 \%$ der erzählten Erinnerungen mit einem Lachen einherging. Dieser Befund bestätigte zahlreiche Ergebnisse zur Erheiterung (siehe z.B. Ruch und Hofmann 2012). Erstaunlicherweise war Erheiterung aber nicht die einzige positive Emotion, die eine starke Assoziation zum Lachen hatte: Schadenfreude löste auch in über $70 \%$ der Geschichten ein Lachen aus und ging mit intensiveren mimischen Ausdrücken einher. Während sich die Häufigkeit und Intensität des Lächelns und Lachens bei Erheiterung und Schadenfreude nicht statistisch unterschieden, waren sie jedoch beide intensiver als viele der anderen positiven Emotionen (zum Beispiel Zufriedenheit oder Dankbarkeit). Die Studie konnte weiter zeigen, dass Schadenfreudeausdrücke öfter mimisch reguliert wurden als andere Ausdrücke positiver Emotionen (Hofmann u.a. 2014) - wobei die Marker der Regulation und deren Dynamik aber nicht im Detail untersucht wurden.

Dies war unseres Wissens die erste empirische Studie, welche in einer natürlichen Auslösebedingung (im Gegensatz zu Studien mit gestellten Emotionsausdrücken ${ }^{5}$ ) zeigen konnte, dass auch Schadenfreude (nicht nur Erheiterung) eine starke Assoziation zum Lachen hat. Im vorliegenden Artikel sollen nun diese mimischen Ausdrücke der Schadenfreude genauer analysiert werden.

In einem ersten Schritt wird die Ähnlichkeit der Schadenfreude-Ausdrücke im Vergleich zu den Ausdrücken der anderen positiven Emotionen untersucht. Dazu werden die distinkten Klassen von kodierten mimischen Ausdrücken in einer Ähnlichkeitsmatrix betrachtet. Es soll untersucht werden, wie ähnlich (oder unähnlich) die Schadenfreude in Bezug auf die Auslösung verschiedener Arten von Lächeln und Lachen im Vergleich zu 15 anderen positiven Emotionen ist (Fragestellung 1).

In einem zweiten Schritt werden die erzählten Erinnerungen zur Schadenfreude einer detaillierten Kodierung mit dem Facial Action Coding System (FACS; Ekman, Friesen und Hager 2002) unterzogen, welche über die Kodierungen in vorhergehenden Analysen herausgeht. Alle Aktionseinheiten werden im Zeitverlauf detailliert kodiert, um so die Abfolgen mimischer Ausdrücke und dabei insbesondere auch die Rolle von Regulationsmarkern zu untersuchen. Dabei werden die drei oben aufgestellten Hypothesen genauer untersucht (Fragestellung 2). 


\section{Methodisches Vorgehen und Ergebnisse}

\subsection{Fragestellung 1: Die Ähnlichkeit von mimischen Schadenfreudeaus- drücken im Vergleich zu mimischen Ausdrücken anderer positiver Emotionen}

Die Stichprobe setzte sich aus 22 Erwachsenen (10 Männern und 12 Frauen) zusammen, welche im Durchschnitt 36,25 Jahre alt waren (Standardabweichung $=13,02$, Min = 19, Max $=60$ ). Die Personen wurden in altershomogene Gruppen aufgeteilt (drei bis vier Personen), in denen sie über ihre Emotionserinnerungen sprachen (siehe Hofmann u.a. 2014). Die mimischen Reaktionen und lautlichen Äußerungen wurden mit zwei Kameras aufgenommen, welche hinter den Personen aufgestellt waren. Die Personen wurden dabei frontal (Kopf und Schulterbereich) bei hoher Auflösung und mit Ton erfasst. Die Videosequenzen wurden mittels dem Facial Action Coding System kodiert.

Das Facial Action Coding System ist ein anatomisch orientiertes Verfahren, mit welchem jede visuell wahrnehmbare Veränderung in der Mimik erfasst werden kann. Dabei bezeichnet jede unterscheidbare Veränderung im Gesicht eine Action Unit (AU, Aktionseinheit; Ekman, Friesen und Hager 2002) und ist mit einer arbiträren Nummer versehen. Das FACS erlaubt eine objektive, reliable und valide Erfassung sämtlicher Bewegungen im Gesicht und ist in der vorliegenden Analyse sowohl für die Unterscheidung verschiedener Ausdrücke des Lächelns und Lachens als auch für das Auffinden von Regulations-Markern relevant. Das FACS liefert Angaben zu Minimalkriterien der Erfassung einer Veränderung und bietet Hilfestellung zur Abgrenzung verschiedener Aktionseinheiten. Dabei findet die Erfassung der Daten ohne Bedeutungszuordnung statt: Interpretationen werden erst nach dem Kodierungsprozess vorgenommen. Ekman, Friesen und Ancoli (1980) sowie Ruch (1995) weisen die Korrelation zwischen subjektiver Befindlichkeit in Selbstberichten und FACSDaten nach, was für die Validität der Interpretationen kodierter Mimik spricht.

Für die erste Analyse zur Ähnlichkeit der Schadenfreude im Vergleich zu den anderen 15 positiven Emotionen in der Auslösung verschiedener Lächeln und Lachen wurden alle Lächeln und Lachen mit dem Facial Action Coding System auf dem Höhepunkt des Lächelns oder Lachens (Apex) kodiert (zu diesem Verfahren siehe Hofmann u.a. 2014). Die Manifestationen des Lächelns und Lachens wurden in der Folge vier Kategorien zugeordnet: Duchenne-Lächeln und -Lachen versus non-Duchenne-Lächeln und -Lachen. Diese vier distinkten Kategorien von Lächeln und Lachen wurden in einer Euklidischen Distanzmatrix verwendet, um die Ähnlichkeit der Emotionen in der Auslösung der vier Display-Kategorien zu untersuchen. Abb. 4 zeigt die graphische Darstellung der Ergebnisse der Distanzmatrix. 


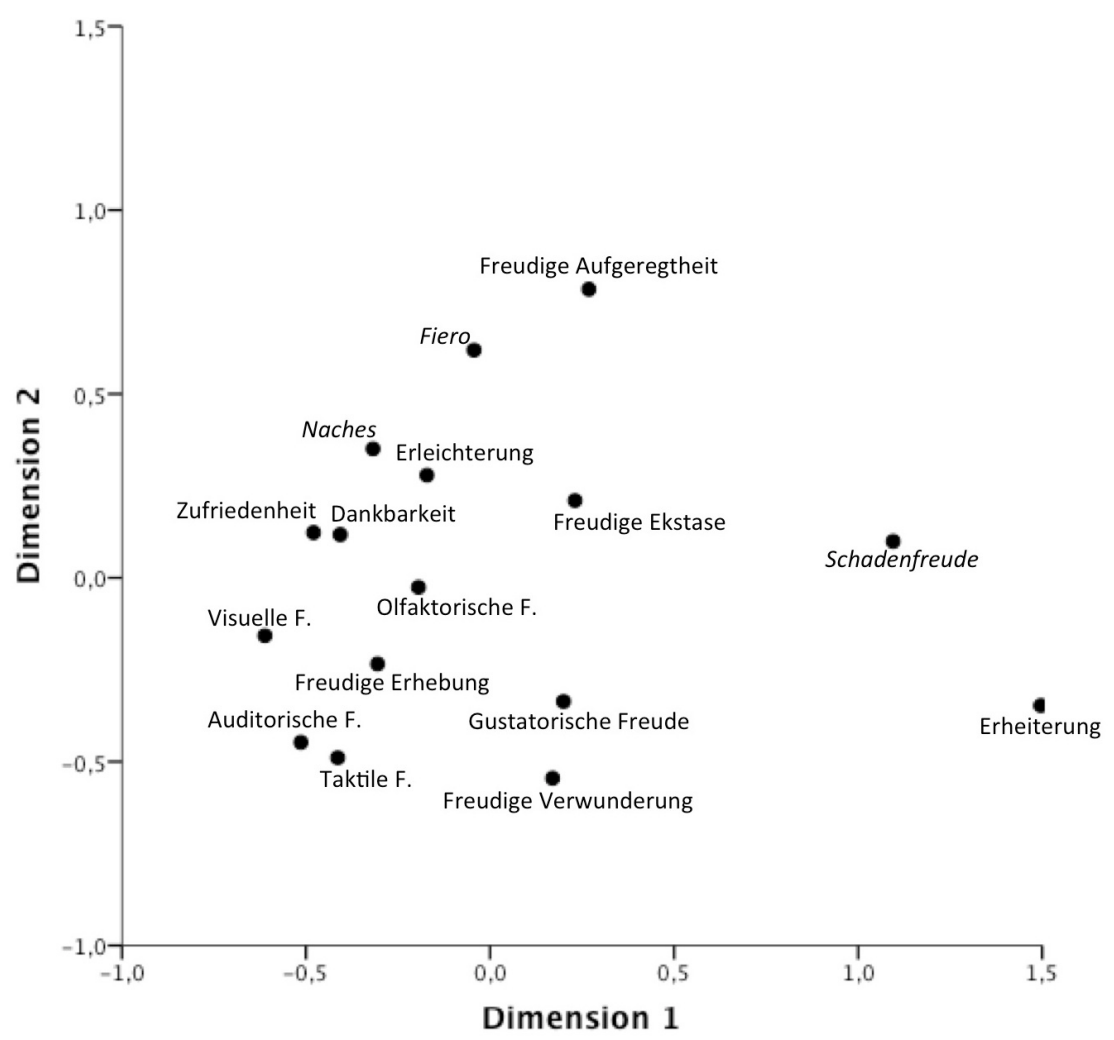

Abb. 4: Euklidisches Distanzmaß (Unähnlichkeitsmatrix) zu den Ähnlichkeiten von 16 positiven Emotionen im Ausdruck von vier distinkten Typen des Lächelns und Lachens.

Abb. 4 ist wie eine geographische Karte zu lesen: Punkte auf der Karte, die näher beieinander liegen, bedeuten größere Ähnlichkeit im Auftreten der distinkten Typen von Lachen und Lächeln. So sind zum Beispiel Zufriedenheit und Dankbarkeit die beiden positiven Emotionen, die sich im Ausdruck von Lächeln und Lachen am ähnlichsten waren (der Unähnlichkeitswert betrug nur 0,07, wobei ein Wert von 0 bedeuten würde, dass die beiden Emotionen gleich sind). Auch die sensorischen Freuden, freudige Erhebung, und Naches (Stolz gegenüber anderen Personen) zeigten ähnliche Ausdrucksmuster (mit Unähnlichkeitswerten kleiner als 0,30). Die größte Unähnlichkeit bestand zwischen den sensorischen Freuden, Erheiterung und Schadenfreude (die letzteren beiden unterschieden sich am stärksten von den fünf sensorischen Freuden). Schadenfreude war der Erheiterung am ähnlichsten, aber beide Emotionen unterschieden sich klar von den verbleibenden 14 Emotionen im Auslösen von Lachen und Lächeln. Die Abbildung zeigt, dass sich die Schadenfreude in der Auslösung von Lachen und Lächeln klar von den anderen 15 Emotionen unterschied. Dieses Ergebnis legt nahe, dass sich, obwohl Schadenfreude mit Duchenne-Lächeln und -Lachen einhergehen kann, die Auftretenshäufigkeiten der verschiedenen 
Kategorien von den anderen Emotionen klar unterscheiden lassen. Schadenfreude und Erheiterung scheinen eine spezielle Rolle einzunehmen, da sie sich stärker von den anderen freudvollen Emotionen unterscheiden.

\subsection{Fragestellung 2: FACS-Detailanalyse von geschilderten Schadenfreude-Erlebnissen}

Für die zweite Analyse wurden alle verfügbaren Videoclips zur Schadenfreude einer weiteren detaillierten Kodierung durch das FACS unterzogen. Von 17 Personen war ein Videoclip verfügbar, auf welchem die Person eine Erinnerung an ein Schadenfreude-Erlebnis berichtete. Die anderen Videos waren aufgrund von technischen Problemen nicht verwendbar. Die 17 Videoclips wurden von zwei zertifizierten FACS-Experten kodiert. Dabei wurden alle mimischen Veränderungen berücksichtigt, die in Reaktion auf das Schadenfreude-Erlebnis auftraten. Mimische Reaktionen auf Äußerungen der anderen Gruppenmitglieder wurden von der Analyse ausgeschlossen.

Beim Erfassungsprozess wurde die empfohlene Kodierprozedur (Ekman, Friesen und Hager 2002) eingehalten. Zuerst wurde die zu kodierende Sequenz angeschaut und das Vorkommen von Aktionseinheiten eingeschätzt. Danach wurden Minimalkriterien der involvierten Aktionseinheiten überprüft und sichergestellt, dass sich die kodierten Aktionseinheiten nicht ausschließen (aufgrund gegenläufiger muskulärer Kontraktionen können bestimmte Bewegungen nicht gleichzeitig auftreten). Nach dem Prüfen subtiler Differenzen zwischen möglichen Aktionseinheiten wurde die endgültige Kodierung festgelegt. Bei sämtlichen Aktionseinheiten wurden sowohl die Intensität und Frequenz als auch die Dauer kodiert. Insgesamt konnten 17 Videos einer Detailkodierung unterzogen werden.

Abb. 5 zeigt die detaillierte Kodierung eines Videoclips, in welchem eine Person ihr Schadenfreude-Erlebnis erzählt. Abb. 5 zeigt alle mimischen Marker, die während des Erzählens der Schadenfreude-Erinnerung aufgetreten sind. Dabei ist jeder mimische Marker mit einer von fünf Intensitätsstufen versehen und nach dem Aktionseinheiten im FACS benannt (AktionsEinheit = AU). Die geringste Intensität ist „A“ (dabei wird die Regung auf dem Gesicht gerade erst sichtbar), die höchste Intensität, die maximal möglich ist, ist mit „E“ verzeichnet. Alle vorkommenden mimischen Marker sind in den Zeilen verzeichnet.

In dieser Schadenfreude-Erinnerung erzählte die betreffende Person, wie sie an der ehemaligen Arbeitsstelle von der vorgesetzten Person schlecht behandelt wurde. Sie erzählte, wie sie wegen der Person die Stelle verlor und es als frustrierend empfand, dass die anderen Mitarbeitenden nicht merkten, wie „falsch“ die vorgesetzte Person war. Dann erfuhr die Person aber (ab Sekunde 20 auf der Abb. 5), dass die Person nun „mit Schimpf und Schande" gefeuert wurde und dass alle in der Firma nun wussten, dass diese Person sich unprofessionell verhalten hatte. Der Zeitverlauf ist auf dem horizontalen Zeitstrahl oben in der Abbildung ersichtlich. 


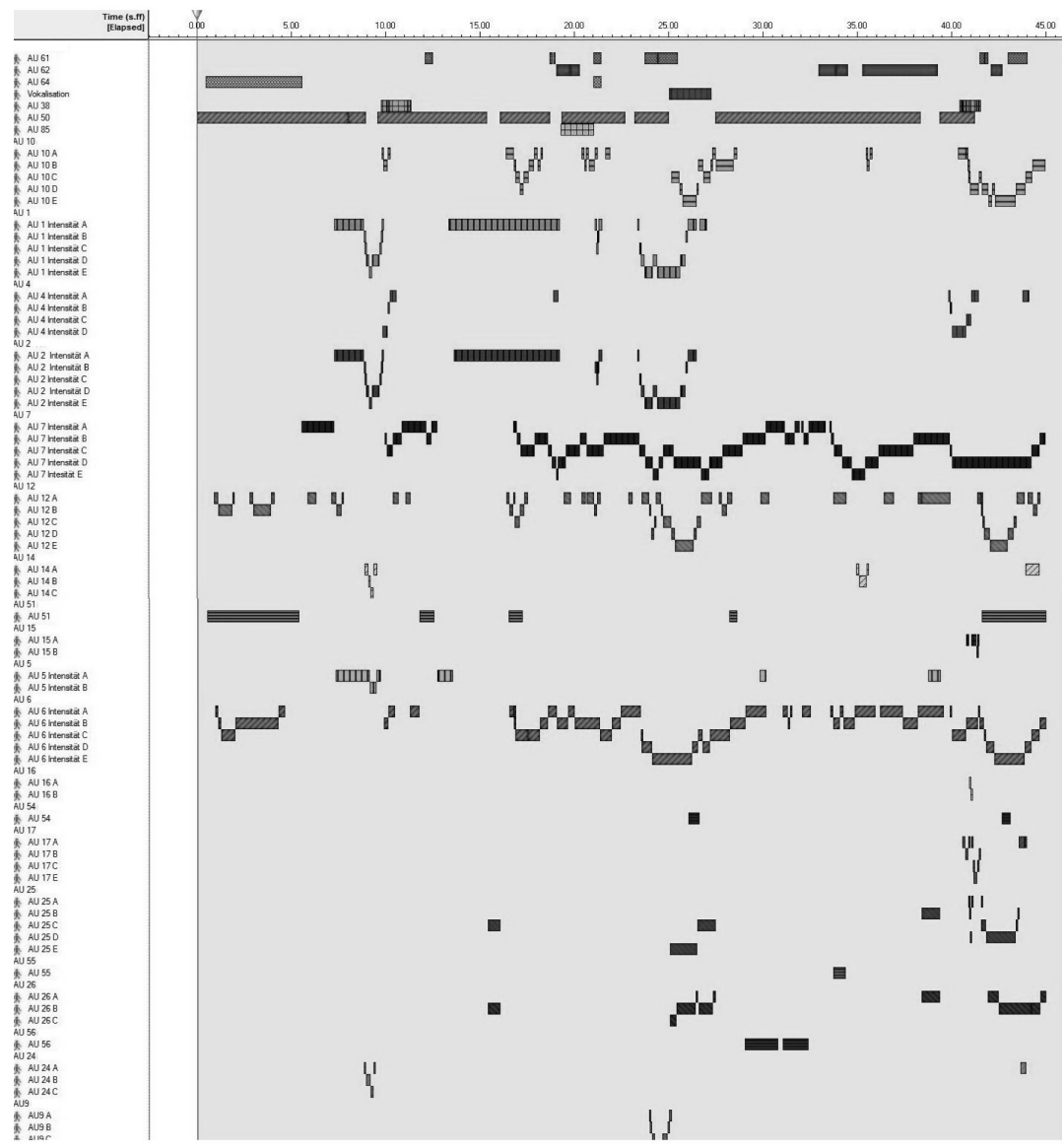

Abb. 5: Detail-FACS-Analyse eines Schadenfreude-Erlebnisses.

Abb. 5 zeigt, dass die Person bei der Rückbesinnung auf ein Schadenfreude-Ereignis mehrere unabhängige Ausdrücke des Lächelns und Lachens zeigt, ${ }^{6}$ wobei einige davon als Duchenne-Lächeln und -Lachen qualifiziert sind (simultanes und symmetrisches Auftreten des Jochbeinmuskels und des äußeren Teiles des Augenringmuskels; AU12 und AU6). Vor allem zum Höhepunkt der Geschichte hin (ca. ab Sekunde 20 in Abb. 5) werden die mimischen Konfigurationen zunehmend komplexer, und das Lachen ist nicht nur von höherer Maximalintensität, sondern auch von längerer Dauer. Zudem weisen die Lachakte zum Höhepunkt der Erzählung hin zusätzliche mimische Marker auf: in der oberen Gesichtshälfte runzelt die Person die Stirn (Aktionseinheit 1 und Aktionseinheit 2; beide bestehend aus der Innervation des $m$. frontalis) und ein Rümpfen der Nase kommt vor. Gegen Ende der Episode treten Regulationsmarker auf, welcher der Aktivierung des Jochbeinmuskels entgegenwirken (Aktionseinheit 15) und die Lippen anspannen. Als die Person in der Erzählung zugibt, starke Schadenfreude empfunden zu haben, wird der Ausdruck der Freude gedämpft (herunter- 
reguliert). Die Person zeigt auch eine Reihe von Kopf- und Augenbewegungen, welche das Gesagte unterstützen (sogenannte „reinforcer“; siehe Ekman 2003). Dieses Beispiel würde die Hypothese 2 unterstützen, welche davon ausgeht, dass Schadenfreude uninhibiert durch ein DuchenneLachen ausgedrückt, in sozialen Situationen (wie in der vorliegenden) aber gedämpft bzw. reguliert wird.

Auf dieselbe Weise wurden die verbleibenden 16 Videoclips mit Schadenfreude-Erinnerungen kodiert und analysiert. Dabei zeigten sich bei den Abfolgen von mimischen Ausdrücken bestimmte Muster.

13 weitere Erzählungen folgten einem ähnlichen Muster wie der Fall, der in Abbildung 5 beschrieben wurde: Die Erzählungen lösten Lächeln und Lachen aus (jeweils mehr als einen Ausdruck), begleitet von verschiedenen Kombinationen von Kopf- und Augenbewegungen und dem Öffnungsgrad der Augen sowie von Regulationsmarkern in verschiedenen Konfigurationen, welche während des Lächelns und Lachens vorkamen. Bei zwei Personen war auffällig, dass das Lächeln oder Lachen permanent gedämpft wurde, durch die Aktivierung der Aktionseinheit 15, welche die Mundwinkel nach unten zieht und so der Aktivierung des Jochbeinmuskels entgegenwirkt. Bei zwei Personen war vor allem eine Aktivierung der Aktionseinheiten 15 und 17 während der Höhepunkte der Geschichten und dem intensiveren Lachen zu beobachten. Weitere Kombinationen von Regulationsmarkern, welche bei den erzählenden Personen wiederholt vorkamen, waren die folgenden: Aktionseinheit 17 und Aktionseinheit 14; Aktionseinheit 14 und 20, bei zwei Personen Aktionseinheit 17 und 24 (presst die Lippen zusammen, basierend auf der Innervation von $\mathrm{m}$. orbicularis oris), ebenso das Zusammenspiel von 14, 15, 17; oder 14, 15, 17, 9 - jeweils zeitgleich zur Anspannung des Jochbeinmuskels (mit oder ohne Beteiligung des m. orbicularis oculi, pars orbitalis). Das gehäufte Auftreten von Regulationsmarkern spricht daher für die Bestätigung der Hypothese 2: die Schadenfreude wird mimisch durch Freude ausgedrückt (Lächeln, Lachen), welche aber im sozialen Kontext entweder gedämpft, maskiert oder reguliert wird. Eine Beeinflussung des Lächelns und Lachens durch sogenannte „smile controls“ (Keltner 1995) konnte in 14 der 17 Clips zu Erlebnissen der Schadenfreude festgestellt werden.

Jedoch wiesen nicht alle Schadenfreude-Clips Lächeln und Lachen auf, welche von Regulationsmarkern begleitet wurden. Bei drei Personen (d.h. in drei Videoclips) zeigte sich zwar Lächeln und Lachen (Duchenne als auch non-Duchenne-Lächeln und Lachen), aber zu keiner Zeit Regulationsmarker (so wie es Hypothese 2 beschreiben würde). In diesen Schilderungen wurde die Schadenfreude offen gezeigt, so wie es Hypothese 2 und Hypothese 3 vorhersagen. Jedoch fehlte die Regulation (Hypothese 2). Diese Lächeln unterschieden sich in der Morphologie auch nicht von dem Lächeln und Lachen der Freude. 


\section{Diskussion}

Die vorliegende Untersuchung zeigt, dass es ein Lachen der Schadenfreude gibt: über $70 \%$ der Erzählungen gingen mit einem Lachen einher (siehe Hofmann u.a. 2014). Dies stimmt mit den Schriften historischer Autoren ebenso wie mit neuen Untersuchungsergebnissen überein.

Der Vergleich der Emotion Schadenfreude mit 15 anderen positiven Emotionen im Ausdruck von vier distinkten Kategorien von Lächeln und Lachen zeigte, dass Schadenfreude der Erheiterung am nächsten war, sich aber auch klar von Erheiterung unterschied. Zudem war die Schadenfreude von den anderen 14 positiven Emotionen stark verschieden.

Unsere Daten legen außerdem nahe, dass Schadenfreude durch das Duchenne-Lachen ausgedrückt wird, wenn es spontan gezeigt wird, und Personen nicht versuchen, dem Ausdruck entgegenzuwirken. In sozialen Situationen, oder wenn die Personen versuchen, sozial akzeptiert zu handeln, legen unsere Daten nahe, dass Personen ihre natürlichen, spontanen Ausdrücke der Schadenfreude regulieren oder dämpfen. Dies drückte sich hauptsächlich durch mimische Marker in der Mundregion aus, welche der Aktivierung des Jochbeinmuskels entgegenwirkten. Dies ist im Einklang mit Befunden von Boecker und Kollegen, welche eine Dissoziation zwischen der Intensität von Emotionseinschätzungen und der mittels EMG gemessenen Muskelanspannung fanden, was auf soziale Erwünschtheit zurückgeführt werden kann.

Die Detailanalyse mit dem Facial Action Coding System zeigte, dass intensiveres Lächeln und Lachen in der Regel gedämpft wurde, und dies meist zum Höhepunkt des Erzählens einer Schadenfreude-Geschichte also zu dem Zeitpunkt, wo die Person zugab, Schadenfreude empfunden zu haben. Dies ist auch kongruent zu den Beschreibungen historischer Autoren, welche in einigen Illustrationen des Schadenfreude-Lachens mimische Marker gezeichnet/dargestellt hatten, welche eine Regulation des Ausdrucks implizierten. Daher unterstützen die vorliegenden Daten vor allem die zweite postulierte Hypothese, dass Schadenfreude durch das Duchenne-Lachen ausgedrückt wird, aber in sozialen Situationen gewissen Ausdrucksregeln unterliegt. Morphologisch ist das Lachen der Schadenfreude also insofern distinkt, dass es im Ausdruck in natürlichen, sozialen Situationen immer von mimischen Regulationsmarkern begleitet ist. Wird die Schadenfreude aber ausgedrückt, wenn eine Person alleine ist oder wenn die Person keinen Grund sieht, den Ausdruck zu dämpfen, sollte das Lachen der Schadenfreude nicht vom Duchenne-Display unterscheidbar sein, so wie es die Hypothesen 2 und 3 vorhersagen.

In der vorliegenden Studie konnten wir keine Hinweise für Hypothese 1 finden, welche postuliert, dass Schadenfreude durch die Emotionsvermischung einer positiven und einer negativen Emotion mimisch dargestellt wird. Obwohl Marker negativer Emotionen auftraten, deuteten sie weniger auf eine Emotionsvermischung als auf regulatorische Funktionen hin. 
Die vorliegende Untersuchung legt nahe, dass Schadenfreude durch die Enkodierenden gedämpft wird (siehe Abb. 3). Ein weiterer interessanter Punkt ist daher die zu erwartende Diskrepanz zwischen dem Selbstbericht der Emotionsstärke der Schadenfreude und der objektiven Messung des Ausdrucks der Schadenfreude. Es ist anzunehmen, dass Personen das Empfinden der Schadenfreude im Selbstbericht als weniger intensiv einschätzen, da diese Emotion nicht sozial erwünscht ist. Der mimische Ausdruck kann daher intensiver sein als die Stärke der empfundenen Emotion im Selbstbericht, da ja die Selbsteinschätzung verzerrt ist (siehe auch Boecker u.a. 2015; Porter u.a. 2014).

Einige Einschränkungen seien erwähnt: In der vorliegenden Studie wurden Personen gebeten, Erinnerungen an Schadenfreude-Erlebnisse zu rekapitulieren, es handelte sich nicht um ein Erleben der Schadenfreude in einem natürlichen Umfeld. Dabei wurden die Emotionen lediglich erinnert und damit wiedererlebt. Daher sollten zukünftige Untersuchungen Ausdrücke von Schadenfreude-Lachen in ökologisch validen Situationen untersuchen und dabei das soziale Umfeld (ob eine Person alleine ist oder nicht) gezielt in die Analysen mit einbeziehen. Zudem ist anzumerken, dass nur wenige Bestrebungen unternommen wurden, Lachen als multimodales Phänomen zu untersuchen - die meisten Studien fokussierten systematisch auf eine Ausdrucksmodalität (Gesicht, Stimme, Atmung, oder Körperbewegungen). Weitere Studien sollten daher das Lächeln und Lachen der Schadenfreude multimodal untersuchen, um alle Arten morphologischer Unterschiede in Gesicht, Stimme und Körperbewegungen zu untersuchen. So könnten in weiterführenden Analysen auch Unterschiede im Lautbild analysiert werden.

\section{Anmerkungen}

* Dem ZIEL-Team (Zurich Interaction and Expression Lab), im Besonderen Dr. Tracey Platt, sei für die Hilfe beim Kodieren der Daten und dem Posieren für die Abbildungen gedankt. Diese Forschung wurde durch ein Projekt der Europäischen Union (7. Rahmenprogramm FP7/2007-2013, Projektvereinbarung n²70780) unterstützt.

1 Siehe z.B. Bachorowski und Owren 2001, 2003; Bryant und Aktipis 2014; Hudenko und Magenheimer 2012; Kipper und Todt 2001, 2003; Kori 1987; Vettin und Todt 2004; Szameitat u.a. 2009, 2011; Urbain u.a. 2014.

2 Siehe z.B. Krumhuber und Manstead 2009.

3 Ausdruckspsychologen und Zeitgenossen, welche verschiedene Arten des Lachens beschrieben haben: Borée (1899), Darwin (1872), Heller (1902), Herland (1938), Huter (1925), Lange (1937), Leonhard (1950), Lersch (1932), Piderit (1867), Rudolph (1903), Schack (1890), Strehle (1954); siehe auch Ruch, Hofmann und Platt (2013) für eine Übersicht.

4 Unter Regulierung wird in diesem Fall die Aktivierung von Muskeln verstanden, welche dem freien Ausdruck des Lachens oder Lächelns entgegenwirken. Dies 
können zum Beispiel Muskeln der Mundregion sein (siehe z.B. Keltner 1995), die dem Heraufziehen der Mundwinkel beim Lächeln entgegenwirken. Zudem sind Bewegungen wie das Verstecken des Gesichtes in den Händen (oder verdecken des Mundes mit der Hand), wegdrehen, Augen schließen usw. möglich.

Gestellte bzw. geschauspielerte Ausdrücke von verschiedenen Arten des Lachens entsprechen nicht unbedingt dem, wie eine Emotion oder ein Geisteszustand natürlicherweise ausgedrückt werden würde: Erstens haben Menschen (vor allem Schauspieler) die Fähigkeit, Arten von Ausdrücken zu erzeugen, die in natürlichen Kontexten gar nicht vorkommen. Zweitens können Ausdrücke gespielt werden, um bestimmte kommunikative Funktionen zu übernehmen („fictive emotional communications" siehe Gosselin, Kirouac und Doré 1997). Gestellte Ausdrücke repräsentieren stereotypische Ausdrücke, die mit hoher Wahrscheinlichkeit richtig entschlüsselt (dekodiert) werden können, nicht aber die natürlichen Ausdrücke widerspiegeln (siehe auch Douglas-Cowie u.a. 2003, für eine Diskussion zu gestellten Emotionsausdrücken und deren Grenzen und Möglichkeiten).

Sobald ein Reaktionsmuster (z.B. Duchenne-Display) durch einen neuen Stimulus unterbrochen wurde, respektive wieder an Intensität zunahm (z.B. bei durchgehender Aktivierung des Jochbeinmuskels mit unterschiedlichen Intensitätsverlaufskurven), wurden zwei separate Reaktionsmuster kodiert. Aufgrund eines hohen Intensitätsniveaus der Emotion konnte es sein, dass die Aktivierung des Jochbeinmuskels von einem zum nächsten Stimulus erhalten blieb. Nach einer Intensitätssteigerung konnte die Reaktion daher als unabhängig gesehen werden.

\section{Literatur}

Ambadar, Zara, Jeffrey F. Cohn und Lawrence Ian Reed (2009), „All Smiles Are Not Created Equal: Morphology and Timing of Smiles Perceived as Amused, Polite, and Embarrassed/Nervous“. Journal of Nonverbal Behavior 33, 1: 17-34.

Bachorowski, Jo-Anne und Michael J. Owren (2001), „Not All Laughs Are Alike: Voiced but Not Unvoiced Laughter Readily Elicits Positive Affect“. Psychological Science 12, 3: 252-57.

Bachorowski, Jo-Anne und Michael J. Owren (2003), „Sounds of Emotion“. Annals of the New York Academy of Sciences 1000, 1: 244-65.

Bänninger-Huber, Eva (1996). Mimik - Übertragung - Interaktion. Bern: Huber.

Bauminger, Nirit (2004), „The Expression and Understanding of Jealousy in Children with Autism". Development and Psychopathology 16, 1: 157-77.

Boecker, Lea, Katja U. Likowski, Paul Pauli und Peter Weyers (2015), „The Face of Schadenfreude: Differentiation of Joy and Schadenfreude by Electromyography“. Cognition and Emotion 29, 6: 1117-1125.

Borée, Albert (1899), Physiognomische Studien. Stuttgart: Julius Hoffmann.

Brigham, Nancy L., Kimberly A. Kelso, Mark A. Jackson und Richard H. Smith (1997), "The Roles of Invidious Comparisons and Deservingness in Sympathy and Schadenfreude“. Basic and Applied Social Psychology 19, 3: 363-80. 
Bryant, Gregory A. und C. Athena Aktipis (2014), „The Animal Nature of Spontaneous Human Laughter". Evolution and Human Behavior 35, 4: 327-35.

Cikara, Mina und Susan T. Fiske (2013), „Their Pain, Our Pleasure: Stereotype Content and Schadenfreude“. Annals of the New York Academy of Sciences 1299, 1: 52-59.

Darwin, Charles (1872). The expression of the emotions in man and animals. London: John Murray.

Douglas-Cowie, Ellen, Nick Campbell, Roddy Cowie und Peter Roach (2003), „Emotional Speech: Towards a New Generation of Databases“. Speech Communication 40, 1-2: 33-60.

Dvash, Jonathan und Simone G. Shamay-Tsoory (2011), „Envy and Schadenfreude: The Neural Correlates of Competitive Emotions“. In: Richard Ebstein, Simone Shamay-Tsoory und Soo Hong Chew (Hrsg.), From DNA to Social Cognition. Hoboken, NJ: John Wiley \& Sons: 139-55.

Ekman, Paul (1985), Telling lies. Clues to deceit in the marketplace, politics, and marriage. New York: W.W. Norton.

Ekman, Paul (1994), „Strong Evidence for Universals in Facial Expressions: A Reply to Russell's Mistaken Critique“. Psychological Bulletin 115, 2: 268-87.

Ekman, Paul (2003), „Sixteen enjoyable emotions“. Emotion Researcher 18, 2: 6-7.

Ekman, Paul, Richard J. Davidson und Wallace V. Friesen (1990), „The Duchenne Smile: Emotional Expression and Brain Physiology: I"“. Journal of Personality and Social Psychology 58, 2: 342-53.

Ekman, Paul und V. Friesen (1982), „Felt, False, and Miserable Smiles“. Journal of Nonverbal Behavior 6, 4: 238-58.

Ekman, Paul, Wallace V. Friesen und Sonia Ancoli (1980), „Facial Signs of Emotional Experience“. Journal of Personality and Social Psychology 39, 6: 1125-34.

Ekman, Paul, Wallace V. Friesen und Joseph C. Hager (2002), Facial Action Coding System: A technique for the measurement of facial movement. Palo Alto: Consulting Psychologists Press.

Ekman, Paul, Joseph C. Hager und Wallace V. Friesen (1981), „The Symmetry of Emotional and Deliberate Facial Actions“. Psychophysiology 18, 2: 101-6.

Ekman, Paul und Erika L. Rosenberg (2005), What the face reveals: Basic and applied studies of spontaneous expression using the facial action coding system (FACS). New York: Oxford University Press.

Feather, N. T. (2008), „Perceived Legitimacy of a Promotion Decision in Relation to Deservingness, Entitlement, and Resentment in the Context of Affirmative Action and Performance“. Journal of Applied Social Psychology 38, 5: 1230-54.

Feather, N. T. und Rebecca Sherman (2002), „Envy, Resentment, Schadenfreude, and Sympathy: Reactions to Deserved and Undeserved Achievement and Subsequent Failure“. Personality and Social Psychology Bulletin 28, 7: 953-61.

Frank, Mark G. und Paul Ekman (1993), „Not All Smiles Are Created Equal: The Differences between Enjoyment and Non-enjoyment Smiles“. Humor: International Journal of Humor Research 6, 1: 9-26.

Fridlund, Alan J. (1991), „Sociality of Solitary Smiling: Potentiation by an Implicit Audience“. Journal of Personality and Social Psychology 60, 2: 229-240. 
Gosselin, Pierre, Gilles Kirouac und François K. Doré (1997), „Components and Recognition of Facial Expression in the Communication of Emotion by Actors“. In: Paul Ekman und Erika L. Rosenberg (Hrsg.), What the Face Reveals: Basic and Applied Studies of Spontaneous Expression Using the Facial Action Coding System. New York: Oxford University Press: 239-267.

Hareli, Shlomo und Weiner, Bernard (2002), „Dislike and envy as antecedents of pleasure at another's misfortune". Motivation and Emotion 26: 257-277.

Harris, Christine R. und Nancy Alvarado (2005), „Facial Expressions, Smile Types, and Self-Report during Humour, Tickle, and Pain“. Cognition and Emotion 19, 5: 655-69.

Heller, Hermann Vincenz (1902), Grundformen der Mimik des Antlitzes. Wien: Anton Schroll.

Herland, Leo (1938), Gesicht und Charakter. Handbuch der praktischen Charakterdeutung. Wien: Saturn.

Hess, Ursula und Robert E. Kleck (1990), „Differentiating Emotion Elicited and Deliberate Emotional Facial Expressions“. European Journal of Social Psychology 20, 5: 369-85.

Hofmann, Jennifer (2014), „Intense or Malicious? The Decoding of Eyebrow-Lowering Frowning in Laughter Animations Depends on the Presentation Mode". Frontiers in Psychology 5 (November).

Hofmann, Jennifer, Tracey Platt und Willibald Ruch (unter Begutachtung), „More than amusement: Laughter and smiling in positive emotions“. Manuskript unter PeerReview.

Hofmann, Jennifer, Willibald Ruch und Tracey Platt (2012), „The en- and decoding of schadenfreude laughter. Sheer joy expressed by a Duchenne laugh or emotional blend with a distinct morphological expression?". Proceedings of the Interdisciplinary Workshop on Laughter and other Non-Verbal Vocalisations in Speech, Dublin, Ireland, 26-27. URL: http://www.coli.uni-saarland.de/conf/laughter-12/files/ abstract_hofmann_et_al.pdf.

Hudenko, William J. und Michael A. Magenheimer (2012), „Listeners Prefer the Laughs of Children with Autism to Those of Typically Developing Children“. Autism 16, 6: 641-55.

Huter, Carl (1925/1985), Physiognomik und Mimik. Schwaig bei Nürnberg: Carl Huter. Indersmitten, Tim und Ruben C. Gur (2003), „Emotion Processing in Chimeric Faces: Hemispheric Asymmetries in Expression and Recognition of Emotions“. The Journal of Neuroscience 23, 9: 3820-25.

Keltner, Dacher (1995), „Signs of Appeasement: Evidence for the Distinct Displays of Embarrassment, Amusement, and Shame“. Journal of Personality and Social Psychology 68, 3: 441-54.

Keltner, Dacher und George A. Bonanno (1997), „A Study of Laughter and Dissociation: Distinct Correlates of Laughter and Smiling during Bereavement“. Journal of Personality and Social Psychology 73, 4: 687-702.

Keltner, Dacher, Paul Ekman, Gian C. Gonzaga und Jennifer S. Beer (2003), „Facial expression of emotion“. In: Richard, Davidson, Klaus Scherer und H. Hill Goldsmith (Hrsg.), Handbook of affective science. London: Oxford University Press: 415-432.

Kipper, Silke und Dietmar Todt (2001), „Variation of Sound Parameters Affects the Evaluation of Human Laughter“. Behavior 138, 9: 1161-78. 
Kipper, Silke und Dietmar Todt (2003a), „Dynamic-Acoustic Variation Causes Differences in Evaluations of Laughter". Perceptual and Motor Skills 96, 3: 799-809.

Kipper, Silke und Dietmar Todt (2003b), „The Role of Rhythm and Pitch in the Evaluation of Human Laughter“. Journal of Nonverbal Behavior 27, 4: 255-72.

Kori, Shiro (1987), „Perceptual Dimensions of Laughter and Their Acoustic Correlates“. Proc. Intern. Confer. Phonetic Sciences Tallinn 4: 255-258.

Krumhuber, Eva und Arvid Kappas (2005), „Moving Smiles: The Role of Dynamic Components for the Perception of the Genuineness of Smiles“. Journal of Nonverbal Behavior 29, 1: 3-24.

Krumhuber, Eva und Antony S. Manstead (2009), „Can Duchenne Smiles Be Feigned? New Evidence on Felt and False Smiles“. Emotion 9, 6: 807-20.

Krumhuber, Eva, Antony S. Manstead, Darren Cosker, Dave Marshall, Paul L. Rosin und Arvid Kappas (2007), „Facial Dynamics as Indicators of Trustworthiness and Cooperative Behavior". Emotion 7, 4: 730-35.

Lange, Fritz (1937), Die Sprache des menschlichen Antlitzes. München: J. F. Lehmann.

Leonhard, Karl (1950/1997), „Der menschliche Ausdruck in Mimik, Gestik und Phonik“. In: Helmut Beckmann und Ernst Franzek (Hrsg.), Der menschliche Ausdruck in Mimik, Gestik und Phonik. 3. Auflage. Würzburg: Klinik und Poliklinik für Psychiatrie und Psychotherapie.

Lersch, Philipp (1951), Gesicht und Seele: Grundlinien einer mimischen Diagnostik. München: Reinhardt.

Matsumoto, David, Dacher Keltner, Michelle N. Shiota, Mark. G. Frank und Maureen O'Sullivan (2008), „What's in a face? Facial expressions as signals of discrete emotions“. In: Michael Lewis, Jeannette M. Haviland und Lisa Feldman Barrett (Hrsg.), Handbook of emotions. New York: Guilford: 211-234.

Paulus, Andrea und Dirk Wentura (2014), „Threatening Joy: Approach and Avoidance Reactions to Emotions Are Influenced by the Group Membership of the Expresser". Cognition and Emotion 28, 4: 656-77.

Piderit, Theodor (1867/1919), Mimik und Physiognomik. Detmold: Verlag der Meyerschen Hofbuchhandlung.

Porter, Stephen, Aisha Bhanwer, Michael Woodworth und Pamela J. Black (2014), „Soldiers of Misfortune: An Examination of the Dark Triad and the Experience of Schadenfreude". Personality and Individual Differences 67: 64-68.

Ruch, Willibald (1990), Die Emotion Erheiterung: Ausdrucksformen und Bedingungen. Habilitationsschrift, Universität Düsseldorf.

Ruch, Willibald (1993), „Exhilaration and Humor“. In: Michael Lewis und Jeannette M. Haviland (Hrsg.), The handbook of emotions. New York: Guilford: 605-616.

Ruch, Willibald (1995), ,,Will the Real Relationship between Facial Expression and Affective Experience Please Stand up: The Case of Exhilaration“. Cognition and Emotion 9, 1: 33-58.

Ruch, Willibald (1997), „State and Trait Cheerfulness and the Induction of Exhilaration: A FACS Study“. European Psychologist 2, 4: 328-41.

Ruch, Willibald und Paul Ekman (2001), „The expressive pattern of laughter“. In: Alfred W. Kaszniak (Hrsg.), Emotion, qualia, and consciousness. Tokyo: Word Scientific Publisher: 426-443. 
Ruch, Willibald, Jennifer Hofmann und Tracey Platt (2013), „Investigating Facial Features of Four Types of Laughter in Historic Illustrations“. The European Journal of Humour Research 1, 1: 99-118.

Ruch, Willibald und Markus Stevens (1995), „The Differential Effects of Nitrous Oxide on Mood Level: The Role of Trait-Cheerfulness“. Vortrag am 7th Meeting of the International Society for the Study of Individual Differences - ISSID, July 15-19, 1995, Warschau, Polen.

Ruch, Willibald und Lisa Wagner (in diesem Heft), „Attribute des Lachens: Ein lexikalischer Ansatz". Zeitschrift für Semiotik 37, 1-2: 109-127.

Rudolf, Heinrich (1903), Der Ausdruck der Gemütsbewegungen des Menschen. Dresden: Gerhard Küthmann.

Schack, Sophus Peter Lassenius (1890), Physiognomische Studien. Jena: Hermann Costenoble.

Schmidt, Karen L., Zara Ambadar, Jeffrey F. Cohn und L. Ian Reed (2006), „Movement Differences between Deliberate and Spontaneous Facial Expressions: Zygomaticus Major Action in Smiling“. Journal of Nonverbal Behavior 30, 1: 37-52.

Schmidt, Karen L., Sharika Bhattacharya und Rachel Denlinger (2009), „Comparison of Deliberate and Spontaneous Facial Movement in Smiles and Eyebrow Raises". Journal of Nonverbal Behavior 33, 1: 35-45.

Smith, Richard H., Terence J. Turner, Ron Garonzik, Colin W. Leach, Vanessa UrchDruskat und Christine M. Weston (1996), „Envy and Schadenfreude“. Personality and Social Psychology Bulletin 22, 2: 158-68.

Strehle, Hermann (1954/1966), Mienen, Gesten und Gebärden: Analyse des Gebahrens. München: Reinhardt.

Sumitsuji, Noboru (1967). „Electromyographic studies on the facial expression“. Psychiatria et Neurologia Japonica 69, 10: 1101-1119.

Szameitat, Diana P., Kai Alter, André J. Szameitat, Chris J. Darwin, Dirk Wildgruber, Susanne Dietrich, und Annette Sterr (2009), ,Differentiation of Emotions in Laughter at the Behavioral Level“. Emotion 9, 3: 397-405.

Szameitat, Diana P., Kai Alter, André J. Szameitat, Dirk Wildgruber, Annette Sterr und Chris J. Darwin (2009), „Acoustic Profiles of Distinct Emotional Expressions in Laughter". The Journal of the Acoustical Society of America 126, 1: 354-66.

Szameitat, Diana P., Chris J. Darwin, André J. Szameitat, Dirk Wildgruber und Kai Alter (2011), „Formant Characteristics of Human Laughter". Journal of Voice 25, 1 : 32-37.

Urbain, Jérôme, Hüseyin Çakmak, Aurélie Charlier, Maxime Denti, Thierry Dutoit und Stéphane Dupont (2014), „Arousal-Driven Synthesis of Laughter“. IEEE Journal of Selected Topics in Signal Processing 8, 2: 273-84.

Van Dijk, Wilco, Jaap Ouwerkerk, Sjoerd Goslinga und Myrke Nieweg (2005), „Deservingness and Schadenfreude“. Cognition and Emotion 19, 6: 933-39.

Van Dijk, Wilco W., Guido M. van Koningsbruggen, Jaap W. Ouwerkerk und Yoka M. Wesseling (2011), „Self-Esteem, Self-Affirmation, and Schadenfreude“. Emotion 11, 6: 1445-49.

Vettin, Julia und Dietmar Todt (2004), „Laughter in Conversation; Features of Occurrence and Acoustic Structure“. Journal of Nonverbal Behavior 28, 2: 93-115. 
Wildgruber, Dirk, Diana P. Szameitat, Thomas Ethofer, Carolin Brück, Kai Alter, Wolfgang Grodd und Benjamin Kreifelts (2013), „Different Types of Laughter Modulate Connectivity within Distinct Parts of the Laughter Perception Network". PLoS ONE 8, 5: e63441.

Dr. Jennifer Hofmann

Psychologisches Institut, Abteilung für Persönlichkeitspsychologie und Diagnostik Universität Zürich

Binzmühlestrasse 14/7

$\mathrm{CH}-8050$ Zürich

E-Mail:j.hofmann@psychologie.uzh.ch

Prof. Dr. Willibald Ruch

Psychologisches Institut, Abteilung für Persönlichkeitspsychologie und Diagnostik Universität Zürich

Binzmühlestrasse 14/7

CH-8050 Zürich

E-Mail:w.ruch@psychologie.uzh.ch 